\title{
Human hepatocyte transplantation for liver disease: current status and future perspectives
}

\author{
V lansante ${ }^{1}$, R R Mitry ${ }^{1}$, C Filippi ${ }^{1}$, E Fitzpatrick ${ }^{1}$ and A Dhawan ${ }^{1}$
}

Liver transplantation is the accepted treatment for patients with acute liver failure and liver-based metabolic disorders. However, donor organ shortage and lifelong need for immunosuppression are the main limitations to liver transplantation. In addition, loss of the native liver as a target organ for future gene therapy for metabolic disorders limits the futuristic treatment options, resulting in the need for alternative therapeutic strategies. A potential alternative to liver transplantation is allogeneic hepatocyte transplantation. Over the last two decades, hepatocyte transplantation has made the transition from bench to bedside. Standardized techniques have been established for isolation, culture, and cryopreservation of human hepatocytes. Clinical hepatocyte transplantation safety and short-term efficacy have been proven; however, some major hurdles-mainly concerning shortage of donor organs, low cell engraftment, and lack of a long-lasting effect—need to be overcome to widen its clinical applications. Current research is aimed at addressing these problems, with the ultimate goal of increasing hepatocyte transplantation efficacy in clinical applications.

O rthotopic liver transplantation (OLT) is currently considered the gold standard treatment for end-stage liver disease, acute liver failure, and liver-based metabolic disorders, and is the only intervention with proven clinical benefits and long-lasting effects. However, the shortage of donors limits the number of patients who may benefit from OLT and means that there is a significant waiting list and high mortality. OLT has several disadvantages, including the risks of complications related to surgery, the high cost of the procedure, and the need for lifelong immunosuppression. Theoretically, a potential alternative to liver transplantation is hepatocyte transplantation (HT), in which transplanted cells provide the missing/impaired hepatic function once engrafted into the recipient's liver. Mature hepatocytes are considered the most obvious cell type for liver cell transplantation since they are the major functional components of the liver. In principle, hepatocyte transplantation has several advantages over OLT: (i) it is less invasive and less expensive not involving a complex surgery; (ii) it can be performed repeatedly if required; (iii) cryopreserved cells isolated from donor livers are available immediately when needed; (iv) native liver remains in place serving as a backup in case of cell graft failure and allowing potential regeneration in patients with acute liver failure (ALF), as well as representing a possible target for future gene therapy in patients with liverbased metabolic disorders; and (v) because this procedure only requires a fraction of the cells isolated from a donor organ, multiple patients can be treated from one donor tissue, thus saving other organs for patients who do require OLT.

The first experimental attempt of hepatocyte transplantation was developed in 1976 (1) to treat the hyperbilirubinemic Gunn rat, an animal model for Crigler-Najjar syndrome type I (CN), and along with other preclinical observations (2-4), it led to the first transplant of autologous hepatocytes in 10 patients with liver cirrhosis in 1992 in Japan with uncertain results (5). Since then, reports have been published of more than 100 patients with liver disease treated by HT worldwide (6). To date, human hepatocytes transplantation has resulted in partial correction of a high number of liver diseases including urea cycle disorders (7-9), factor VII deficiency (10), glycogen storage disease type 1 (11), infantile Refsum's disease (12), phenylketonuria (13), severe infantile oxalosis (14), and acute liver failure (15-18) (Table 1). The level of cell replacement required to achieve physiological benefit varies depending on the disease, and it has been considered around $5-10 \%$ of theoretical liver mass in the transplantations carried out so far. Although encouraging clinical improvements are seen in patients transplanted with allogeneic hepatocytes, longterm efficacy is still hampered by insufficient engraftment/ long-term acceptance of cellular allografts, despite immunosuppression (19). Even though promising, HT still faces limitations. First, there is a limited supply of donor livers from which high-quality hepatocytes can be isolated, as the source is suboptimal livers that are otherwise unsuitable for organ transplantation (20). These cells are often poorly functioning. Second, cryopreservation of hepatocytes results in impaired cell viability after thawing due to freeze-thaw damage (21). Third, cell engraftment is quite poor, estimated to be from 0.1 to $0.3 \%$

'DhawanLab, Paediatric Liver Gl and Nutrition Center and MowatLabs, Institute of Liver Studies, King's College London, Faculty of Life Sciences and Medicine, King's College London, King's College Hospital, London, UK. Correspondence: A. Dhawan (anil.dhawan@kcl.ac.uk)

Received 15 June 2017; accepted 2 October 2017; advance online publication 6 December 2017. doi:10.1038/pr.2017.284 


\section{Hepatocyte transplantation $\mid$ ReView}

Table 1. List of human liver diseases treated with hepatocyte transplantation

\begin{tabular}{l}
\hline Disease \\
\hline Inborn error diseases \\
Alpha1-antitrypsin (34) \\
Crigler-Najjar syndrome type I (41-43) \\
Familial hypercholesterolemia (45) \\
Factor VII deficiency (10) \\
Glycogen storage diseases (11,44) \\
Infantile Refsum's disease (12) \\
Primary oxalosis (14) \\
Phenylketonuria (13,40) \\
Progressive familial intrahepatic cholestasis (Dhawan et al., unpublished \\
data) \\
Urea cycle defects (7-9,36-40) \\
Acute liver failure \\
Drug (16) \\
Viral (16) \\
Idiopathic (15) \\
Mushroom poisoning (18) \\
Acute fatty liver of pregnancy (17)
\end{tabular}

of host liver mass in mice after infusion of $3-5 \%$ of the total recipient liver cells (22). Finally, allogeneic rejection is most probably the main cause for the observed decline of cell graft function within 1 year of HT (19). Although the safety of clinical HT has been very well established, therapeutic benefit has been modest and only temporary so far. This is partially in contrast with very convincing preclinical results obtained in a number of animal models of liver disease (2-4), possibly because these models do not properly mimic the disease in humans. Animals usually develop liver injury within a few days or weeks, whereas in humans the injury is significantly more severe occurring over many decades, thus highlighting a need of more realistic models of liver disease.

\section{PROCEDURES: HEPATOCYTE SOURCE, ISOLATION, QUALITY CONTROL, AND ADMINISTRATION FOR CELL \\ TRANSPLANTATION}

Human hepatocytes are isolated from (i) whole donor livers unsuitable/rejected for OLT, mostly because of severe steatosis, prolonged ischemia time, older, or non-heartbeating donors, or (ii) from liver segments, mainly I and IV, which are available for cell isolation after split liver transplantation. Alternatively, fetal and neonatal livers are being explored as a potential cell source $(23,24)$ as they are currently not considered for OLT and may allow the isolation of very high-quality cells (9). Neonatal-derived hepatocytes, although not fully mature, show activity of some of the main enzymes responsible for hepatic-detoxifying functions (23). Explanted livers from patients with liver-based metabolic disorders have also been considered as a potential source of cells. This concept was adopted from a similar approach used in the so-called "domino" liver transplantation performed since the mid-1990s, in which a liver transplant recipient with a known metabolic defect can donate his explanted native liver for use as liver graft in another patient (25). In a similar way, liver explants from patients with defined metabolic disorders could become a source of hepatocytes, since their missing enzymatic function would be performed by the recipient's native liver, left in place. This approach was used in a 6-year-old boy with severe phenylketonuria transplanted with hepatocytes isolated from a glycogen storage type $1 \mathrm{~b}$ liver explant, with significant reduction of blood phenylalanine levels up to 3 months after transplantation (13).

In order to isolate the hepatocytes, a standardized three-step collagenase perfusion technique originally developed by Berry and Friend and later modified (26-28) is used. First, major hepatic vessels are cannulated and perfused with Hank's buffered salt solution (HBSS) containing EGTA, which is able to chelate calcium ions, thus disrupting the desmosomes, calciumdependent cell-to-cell adhesions. Second, the liver is flushed with plain HBSS to remove any residue of EGTA. Third, the tissue is perfused with a calcium-dependent collagenase solution to digest the extracellular matrix. After breaking of the liver capsule, the released liver cells are filtered and centrifuged at low speed to separate hepatocytes from nonparenchymal cells. Hepatocytes can be used fresh for transplantation or cryopreserved. Several protocols have been described to cryopreserve human hepatocytes, with the best results being achieved cryopreserving $10^{6}-10^{7}$ cells $/ \mathrm{ml}$ in the University of Wisconsin organ preservation solution containing $10 \%$ dimethyl sulfoxide and $5 \%$ glucose, and using a controlled-rate freezer (29). Cryopreserved hepatocytes are then stored at $-140^{\circ} \mathrm{C}$ until required for HT. Hepatocyte isolations for use in clinical HT must be performed under good manufacturing practice (GMP) in an aseptic and accredited units.

Evaluation of hepatocyte quality before transplantation is vital, especially on cryopreserved cells, as it has been shown that freeze-thawing cycles damage mitochondria, affect cell respiration with a dramatic drop of ATP levels (21), and may induce apoptosis (unpublished data). Most centers use trypan blue exclusion test as the main and often only criterion to assess cell quality before transplantation, usually accepting cells with viability equal or higher than $60 \%$ for clinical applications. However, viability assessment does not detect any sign of early apoptosis in the cells or their functions, which should probably be included in the cell quality control to ensure that high-quality hepatocytes are transplanted (30). In keeping with GMP standards, microbiological analysis must be undertaken at each stage of cell preparation and the final product must be exempt from any contamination before release of hepatocytes for cell transplantation.

The most widely used administration route for clinical delivery of HT is intraportal infusion, with the catheter placed in the portal vein or in one of its branches. In newborn babies, the portal vein can be accessed by catheterization of the umbilical 


\section{Review | Iansante et al.}

vein. In older children, a cut-down approach may be used or a laparoscopic/mini laparotomy insertion of a central venous cannula into a tributory of the portal vein. Hepatocytes $(\sim 20-40 \mu \mathrm{M}$ in diameter) move across the sinusoidal vessels and induce transient occlusions in the periportal vascular areas dependent on cell size, followed by restoration of normal blood flow by vascular permeabilization, which allows transplanted cells to reach the liver parenchyma $(31,32)$. Hepatocytes remaining in the portal vessels are cleared by macrophages within $24 \mathrm{~h}$. The number of cells injected is limited by portal pressure, which should be monitored throughout by Doppler ultrasound, and should not exceed $12 \mathrm{~mm} \mathrm{Hg}$ to avoid the risk of portal hypertension (33). Cell dose and infusion rate depend on the patient weight and clinical situation, with the aim of injecting around $5 \%$ of the total liver mass. Most centers consider a dose of $2 \times 10^{8}$ cells per $\mathrm{kg}$ of body weight as the upper safe limit for hepatocyte transplantation. In some situations when a high number of cells is needed to ensure clinical benefits, multiple infusions can be performed. Intrasplenic administration has been suggested as an alternative to intraportal injection in patients with liver cirrhosis, in whom altered liver architecture impacts hepatocyte engraftment (34). The peritoneal cavity represents an attractive administration route as it is easily accessible and may contain a large number of cells. For these reasons, it has been used in patients with acute liver failure $(15,17)$. However, lack of anchorage for the transplanted cells and host immune response result in a rapid decline in transplanted cell viability. A way to overcome this problem is the encapsulation of hepatocytes in alginate microbeads so that immune cells cannot reach them, thus prolonging their survival (35).

\section{INDICATIONS}

Hepatocyte transplantation has been used clinically both in congenital metabolic disorders affecting the liver and acute liver failure (Table 1). The most encouraging outcomes have been shown in liver-based metabolic disorder, where the number of cells needed to compensate for a single gene defect is lower than that for acute liver failure.

\section{Inborn Errors of Liver Metabolism}

Urea cycle defects represent the most common liver-based metabolic disorders treated with hepatocyte transplantation. These diseases are characterized by an incapacity of fully converting ammonia into urea, with hyperammonemic crisis, frequently leading to death. To date, hepatocyte transplantation has been used in different types of urea cycle defects, including ornithine transcarbamylase (OTC) deficiency (7,36-40), argininosuccinate lyase (ASL) deficiency (8), citrullinemia (7), and carbamoylphosphate synthetase (CPS) deficiency $(7,40)$. Although most patients showed clinical benefits, the effects were not maintained over the long term. Soltys et al. recently published findings from two patients with urea cycle defects, who underwent hepatocyte transplantation after preconditioning with irradiation. The two patients, a 4-month-old girl with CPS1 deficiency and a 7-monthold child with OTC deficiency, received 1.25 and $2.0 \times 10^{8}$ viable hepatocytes/kg body weight, respectively. Clinical benefits were seen in the patient with OTC deficiency, however, after around 40 days, the child developed frequent hyperammonemia. Both children received an OLT after a few months (40).

Crigler-Najjar syndrome is due to a defective UDPglucuronosyltransferase (UGT) enzyme determining increased serum-unconjugated bilirubin levels, which can lead to severe brain damage and death. Current treatments include liver transplantation and phototherapy. There are reports of patients who have received up to 7.5 billion hepatocytes, showing up to $50 \%$ reduction in bilirubin and increase in UGT activity. The majority of these patients received an OLT within a year. One patient was transplanted 4 years later (41-43).

Glycogen storage disease type I is due to mutations impairing the activity of glucose-6-phosphatase complex, with abnormal accumulation of glycogen and fat in the liver and kidneys. Hepatocyte transplantation was used in two adults with improved glucose control up to $7-9$ months $(11,44)$.

Other liver-based metabolic diseases shown to improve following hepatocyte transplantation include factor VII (fVII) deficiency, where there was a $70 \%$ reduction in the need for recombinant fVII for 6 months (10), and familial hypercholesterolemia, where autologous ex vivo-corrected hepatocytes were transplanted with not significant clinical improvement, probably due to low efficiency of genetic correction (45). In addition, hepatocyte transplantation has been used to treat patients with alpha1-antitrypsin (34), infantile Refsum's disease (12), primary oxalosis (14), and phenylketonuria $(13,40)$. Safety of the method was confirmed in all these treatments, however, similarly to the other diseases treated, efficacy was not maintained over the long term.

\section{Acute Liver Failure}

Hepatocyte transplantation aims to bridge patients with acute liver failure to recovery through regeneration of their own liver or to OLT in case an organ becomes available. Cell number necessary to provide hepatic function able to replace the injured liver is slightly higher than for metabolic disorders, estimated to be $\sim 10-15 \%$ of liver cell mass (46). Cell transplantation through the portal system is not always possible in these patients, due to frequent coagulopathy issues; therefore, other administration routes have been used, such as intraperitoneally $(15,17)$ or intrasplenic $(16,34)$.

In many cases, hepatocyte transplantation in patients with fulminant liver failure resulted in clinical improvements with reduction in ammonia and bilirubin and hepatic encephalopathy, as already extensively described in other reviews $(34,47)$. However, it is difficult to conclude about the efficacy of this approach without randomized controlled trials.

\section{IMMUNOSUPPRESSION AND IMMUNE RESPONSE TO CELL GRAFT}

There is no consensus regarding the optimum immunosuppressive treatment following HT. Most centers have adapted immunosuppressive protocol used for orthotopic liver transplantation to HT. Common immunosuppressive 


\section{Hepatocyte transplantation Review}

regimens include induction with steroids and calcineurin inhibitors (tacrolimus or cyclosporine) $(8,10,11,42)$. Some centers have also included therapy with anti-interleukine-2 receptor antibodies (basiliximab) (7). Experiences with OLT have highlighted that liver is an immune-privileged organ, leading in some cases to graft tolerance development and possibility of immunosuppression withdrawal. Hepatocyte transplantation has been erroneously considered similar to liver transplantation in terms of immunogenicity and it was initially assumed that hepatocytes would have the same immune privilege as the whole liver. Unfortunately, allogeneic hepatocytes seem to be highly antigenic in vivo unlike the liver (20). This discrepancy between inherent tolerogenicity of whole-liver and isolated hepatocytes may suggest that either other hepatic cells like stellate cells or liver sinusoid endothelial cells contribute to liver tolerance (48) or that hepatocytes lose their tolerogenic potential in an allogeneic environment (49). Rejection of hepatocytes is mediated by both the innate and adoptive immunity, and detailed elucidation of the mechanisms involved will probably improve HT outcome.

The innate immune system plays a critical role after HT, as it has been demonstrated that up to $70 \%$ of transplanted hepatocytes might be eliminated by early phagocytic immune response, without significant differences between allogeneic and syngeneic cell transplantation (50). Different mechanisms seem to be involved in hepatocyte early rejection. First, part of the transplanted cells undergo cytolysis mediated by granulocytes and monocytes activated by surface adhesion proteins expressed on hepatocyte cell membrane, like cadherins, otherwise hidden and inaccessible to them in case of wholeliver transplantation (51). Second, a process first described in islet transplantation and known as "instant blood-mediated inflammatory reaction" (IBMIR) is partly responsible for cell loss. The IBMIR is initiated by tissue factor expressed on hepatocyte cell membrane and is characterized by activation of the coagulation and complement systems, binding of activated platelets to hepatocyte surfaces with formation of clots, and infiltration of polymorphonuclear leukocytes resulting in the loss of transplanted cells $(52,53)$.

In addition to the innate immune response, hepatocytes are also rejected by the adaptive immune system. It has been shown that injecting hepatocytes in T-cell-deficient mice improved cell graft survival, not observed in case of B-deficient, or selectively $\mathrm{CD}^{+}$or $\mathrm{CD}^{+}$T-cell-deficient mice, suggesting that immunologic rejection of allogeneic hepatocytes is mediated primarily by T cells (54). More recent studies showed a lack of donor-specific antibodies, confirming that humoral B-cell-mediated response may be not involved in hepatocyte rejection (38).

\section{IMPROVEMENT IN ENGRAFTMENT/PROLIFERATION OF TRANSPLANTED CELLS}

The outcome of HT is strictly dependent on the level of engraftment of transplanted hepatocytes into the recipient's liver (Figure 1). This concept applies both to acute liver failure and liver-based metabolic diseases. In case of metabolic disorders, the ultimate goal is to achieve a long-lasting level of cell integration allowing a stable expression of the missing protein/enzyme over time. Several strategies have been investigated to increase engraftment and/or proliferation of transplanted hepatocytes. There has been much interest in using the therapy in certain diseases that provide a natural selective advantage for transplanted cells, such as hereditary tyrosinemia type I (55), alpha1-antitrypsin deficiency (56), and Wilson's disease (57). Unfortunately, most liver diseases do not offer a selective advantage to transplanted cells, thus making necessary the development of new strategies to achieve this aim, including genetic modification of donor cells or induced injury to the recipient's liver.

A study aiming to enhance the proliferation capacity of donor hepatocytes before transplantation showed that transfecting freshly isolated murine hepatocytes ex vivo with the nonviral sleeping beauty (SB) transposon vector carrying FoxM1 gene, a known cell-cycle regulator, significantly improved liver repopulation without inducing tumorigenesis (58). Many attempts have been made to precondition the recipient's liver and give a selective advantage to the transplanted cells, mainly partial hepatectomy (59), portal embolization (60), and liver irradiation (40), as suggested by a consensus meeting held in London in 2009, where the main representatives of the active clinical hepatocyte transplant programs identified the main limitations of HT and suggested solutions to overcome them (61).

Partial hepatectomy has been successfully used in several animal models of liver disease and showed significant liver repopulation $(62,63)$. Despite its clinical safety being reported more than two decades ago (45), there are still some doubts about its efficacy in humans. Recently, two patients with Crigler-Najjar syndrome type I underwent partial hepatectomy followed by hepatocyte transplantation. Even though cell graft function was lost in one patient for discontinuation of immunosuppression, the other patient showed a decrease of bilirubin to $\approx 50 \%$ of pretransplant concentrations for more than 6 months. However, he finally underwent liver transplantation since graft functions were completely lost $\sim 600$ days after HT (59).

Another approach to improve donor hepatocyte engraftment is partial embolization of the portal vein. This procedure is routinely used in patients undergoing liver resection in case the remaining liver is too small to ensure hepatic function (64). Portal vein embolization (PVE) is safe, well tolerated by patients, and induces hypertrophy of nonembolized liver lobes. Dagher et al. (60) demonstrated that PVE of $50 \%$ of the liver mass followed by HT in Macaca monkeys resulted in replacement of $10 \%$ of the organ total volume in the nonembolized lobe, and reversible PVE obtained with an absorbable material determined similar results (65). Partial hepatectomy with PVE was used in nonhuman primates and in Watanabe-heritable hyperlipidemic rabbits, an animal model of familial hypercholesterolemia type IIa (FH), 


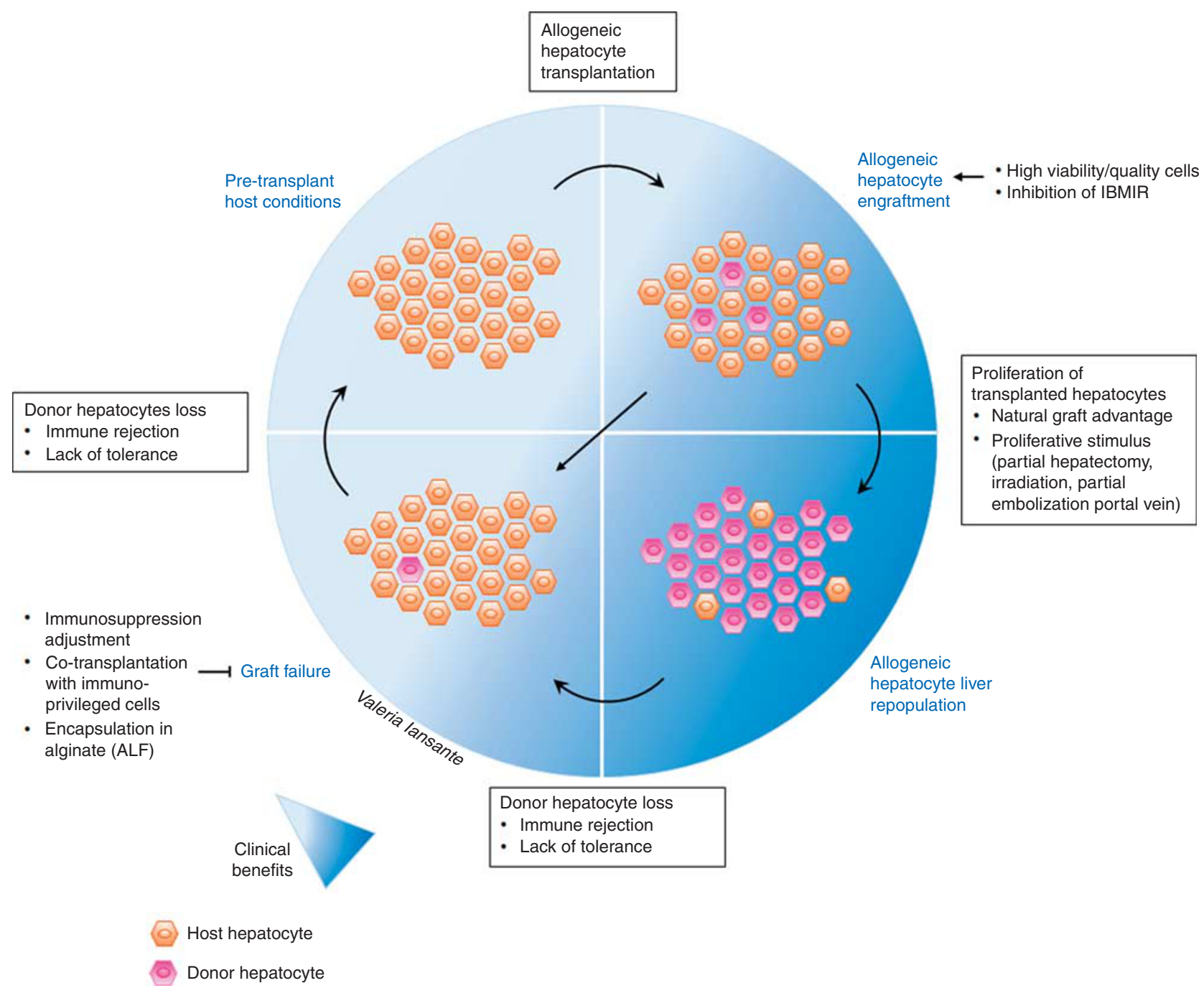

Figure 1. Fate of allogeneic hepatocytes after transplantation. After transplantation, allogeneic hepatocyte engrafts into the recipient's liver. In case of selective advantage over the host cells or in the presence of proliferative stimuli, transplanted hepatocytes repopulate the liver. Immune rejection is the main cause of cell loss and graft failure. Potential ways to improve engraftment and inhibit allogeneic hepatocyte loss are listed. Intensity of blue in the background highlights the level of clinical benefits associated to the different conditions. ALF, acute liver failure.

followed by transplantation of autologous hepatocytes transduced ex vivo with lentiviral vectors $(65,66)$.

Irradiation of the native liver has been investigated as a way to inhibit native hepatocyte proliferation and enhance the engraftment and proliferation of donor hepatocytes. Preclinical studies of HT in the Gunn rat model of Crigler-Najjar syndrome type I, showed that preparative hepatic irradiation on a portion of the liver combined with stimulation of hepatocyte proliferation by administration of hepatocyte growth factor viral vector $24 \mathrm{~h}$ after HT led to a complete correction of the metabolic defect during the 16-week observation period (67). A recent study showed that porcine hepatocytes were transplanted into immunosuppressed nonhuman primates preconditioned with $10 \mathrm{~Gy}$ liver-directed irradiation targeting the right lobe, engrafted, and proliferated constituting up to $15 \%$ of the irradiated lobe (40). Furthermore, irradiation as a host-preconditioning approach was used in three patients treated with HT. Two infants with urea cycle defects preconditioned with irradiation on $1 / 3$ of the liver mass before HT showed early graft loss. Immune rejection was monitored through measurement of $\mathrm{CD} 154^{+}$ T-cytotoxic memory cells ( $\mathrm{TcM})$, shown to predict acute cellular rejection in children with intestine and liver transplantation $(68,69)$. CD154 ${ }^{+} \mathrm{TcM}$ were measured in a 16-h mixed lymphocyte culture, consisting of recipient peripheral blood lymphocytes (PBL) cocultured with donor or third-party HLA-mismatched PBL. If donor lymphocytes were not available, PBL from normal human HLA-matched subjects were used. The immunoreactivity index (IR), represented by the ratio of donor-induced to third-partyinduced $\mathrm{CD} 154^{+} \mathrm{TcM}$, was analyzed every 7 days after cell transplantation and used as a sign of increased risk of rejection when $I R \geq 1.3$. An adult with classical phenylketonuria who received anti-lymphocyte antibody therapy and underwent more frequent immune monitoring/immunosuppression adjustments in addition to the preparative radiation 


\section{Hepatocyte transplantation Review}

treatment, showed multiple clusters of proliferating transplanted hepatocytes and significant decrease of blood phenylalanine levels after HT, although graft loss occurred after follow-up became inconsistent. Despite the encouraging results, the authors of this study highlighted that the level of donor hepatocyte replacement was lower than expected, presumably because HT was performed too early within $24 \mathrm{~h}$ after preconditioning (40).

An additional difficulty in cell engraftment evaluation after HT is the lack of tracking methods to measure the number of transplanted hepatocytes in an accurate and noninvasive way. In preclinical studies, cells can be easily labeled with transfection/transduction of a fluorescent protein or prestained with a dye and monitored over time. However, this approach is not applicable in humans. Hepatocyte labeling with radioactive indium-111 was used in a 5-year-old child with OTC deficiency treated with HT and resulted in a noninvasive analysis of the biodistribution of transplanted hepatocytes (70). Although promising, this technique can be useful only for short-time tracking, being limited by the short half-life of the radioisotope. Labeling with magnetic resonance contrast agents has also been proposed (71) and investigated in preclinical studies (72). In liver-based metabolic disorders, indirect evidence of engraftment after HT can be obtained from levels of metabolic products as a consequence of the introduction of the missing enzyme/protein (e.g., decrease of ammonia in urea cycle defects and bilirubin in Crigler-Najjar patients). However, these measures do not provide reliable information about the exact number of functioning engrafted hepatocytes. Direct evidence of engraftment can be obtained by analyzing liver biopsies for sex chromosome, short tandem repeats, or HLA antigens, if different in a recipient and a donor $(22,73)$. However, biopsies are not always available, being invasive. Furthermore, they only sample a very small proportion of tissue, thus being unrepresentative of the entire liver. An alternative approach to quantify cell engraftment after HT could be the quantification of proteins released in the bloodstream carrying polymorphisms in a donor and recipient detectable by mass spectrometry (e.g., apolipoprotein and haptoglobin). A similar approach may consist of the characterization of exosome cargos released by donor grafts, as shown in a xenogeneic islet transplant model (74). High interest has been recently shown for the development of new methods to measure graft rejection. Since cell-free circulating DNA (cf-DNA) is released into the bloodstream by necrotic and apoptotic cells and can be genotyped and matched to donor cells, it has been successfully used as a marker of early rejection in patients after liver transplantation (75). However, additional studies are required to investigate whether this method can be translated to the detection of rejection in case of cell transplantation, where the number of cells releasing cfDNA is significantly lower than in organ transplantation.

\section{FUTURE DEVELOPMENTS}

The improvement of cell engraftment by host conditioning or donor cell modification, as well as a better understanding of the immune responses involved in hepatocyte rejection are definitely needed in order to overcome the issues limiting HT efficacy. In addition to this, a limited source of hepatocytes and often poor quality of isolated cells still represent an issue for wider clinical applications. Other cell sources, combination therapies, and cell transplantation strategies have been considered to improve HT outcome.

Mesenchymal stromal cells (MSCs) represent an attractive tool for regenerative medicine due to their ability to proliferate and differentiate, as well as their antiinflammatory and immune-privileged status. MSCs exist in many tissues, including bone marrow, umbilical cord, and adipose tissues, and are able to differentiate in mesenchymal tissue cells, i.e., adipocytes, osteoblasts, and chondrocytes. MSCs have recently been isolated from the liver and named liver-derived human MSCs (LHMSCs). Some studies have shown that these cells can be transdifferentiated into hepatocyte-like cells, thus potentially being used for cell transplantation in liver-based disorders $(76,77)$. Although it is still debated whether LHMSCs may efficiently repopulate a host liver and provide adequate hepatic function (78), their anti-inflammatory role is undoubted (79). Furthermore, MSCs derived from bone marrow or umbilical cord and cocultured in vitro with human hepatocytes have shown significant improvement of hepatic functions (80), suggesting that cotransplanting these cells and/or possibly LHMSCs with hepatocytes might ameliorate HT outcome, decreasing inflammation and improving hepatocyte performance.

There has been worldwide interest in the reprogramming of differentiated somatic cells to induced pluripotent stem cells (iPSCs), which can be potentially differentiated into cells belonging to any germ layer, including cells with hepatocytelike morphology and function, known as hepatocyte-like cells (HLCs) (81). Several groups have developed efficient and standardized protocols for the development of iPSC-derived HLCs, some with GMP-approved protocols and reagents. However, HLCs generated by current methods are not fully mature, being phenotypically and functionally more similar to fetal than adult hepatocytes, with low albumin and urea production, and persistent high expression of alpha-fetoprotein, despite showing some CYP450 activity, as well as glycogen and lipid storage activity (82). It is likely that these hurdles regarding iPSC-derived HLC production will be overcome in the near future, with several groups working on this worldwide (83). However, a main safety concern for clinical applications is their tumorigenic potential, which needs to be solved before these cells can be used safely in humans.

In an attempt to decrease the immune response against transplanted allogeneic cells in patients with liver-based metabolic defects and thus improving engraftment, ex vivo genetic correction of hepatocytes for autologous transplantation may be considered. This approach was used more than two decades ago in five patients with familial hypercholesterolemia who underwent partial hepatectomy of the left lateral lobe, followed by hepatocyte isolation, retroviral transduction 


\section{Review $\mid$ Iansante et al.}

of the low-density lipoprotein (LDL) receptor, and autologous transplantation. Although significant and prolonged reductions in LDL cholesterol were demonstrated in three out of five patients, the use of retroviral viruses - unable to transduce nonproliferating cells-drastically impaired clinical efficacy (45). This approach has been recently reconsidered in a porcine model of hereditary tyrosinemia type $\mathrm{I}$, in which ex vivo transduction of hepatocytes with lentiviral vector carrying FAH missing gene was performed followed by autologous transplantation, with very encouraging results (84). This approach would be even more promising if applied to HLC derived from iPSCs, thus decreasing the invasiveness of the method.

Finally, a simple approach to avoid the immune reaction during HT has been proposed for the management of patients with ALF. Human hepatocytes can be encapsulated in alginate microbeads (85), and then transplanted intraperitoneally. This approach would allow transplanted donor hepatocytes to perform all the hepatic functions absent in the failing liver, without the need of immunosuppression as the alginate would be able to protect donor cells from the host immune cells. The technique has been proven successful in a rat model of acute liver injury (35), and has shown encouraging results when used in children with ALF (unpublished). Furthermore, a recent study has shown that alginate microbeads containing hepatocytes can be cryopreserved with some maintenance of hepatic functions after thawing, figuring the possibility of an off-the-shelf product available in an emergency setting typical of fulminant liver failure (86).

\section{CONCLUSION}

The last two decades have seen a considerable development of hepatocyte transplantation, with demonstration of short-term efficacy and safety. However, some hurdles still need to be overcome to widen hepatocyte transplantation clinical applications, mainly regarding isolation of high-quality cells from marginal donor liver, improvement of cell engraftment, and long-lasting effects, as well as optimal immunosuppression regimen identification.

New challenges are needed to improve hepatocyte transplantation efficacy, and include mainly the identification of new cell sources, which could overcome liver donor scarcity, and new approaches to decrease the impact of immune response against transplanted cells, hence improving cell engraftment in liver-based metabolic disorders.

\section{STATEMENT OF FINANCIAL SUPPORT}

The authors acknowledge "MowatLabs" for financial support.

Disclosure: The authors declare no conflict of interest.

\section{REFERENCES}

1. Matas AJ, Sutherland DE, Steffes MW, et al. Hepatocellular transplantation for metabolic deficiencies: decrease of plasma bilirubin in Gunn rats. Science 1976;192:892-4.
2. Sutherland DE, Numata M, Matas AJ, Simmons RL, Najarian JS. Hepatocellular transplantation in acute liver failure. Surgery 1977;82: 124-32.

3. Yoshida Y, Tokusashi Y, Lee GH, Ogawa K. Intrahepatic transplantation of normal hepatocytes prevents Wilson's disease in Long-Evans cinnamon rats. Gastroenterology 1996;111:1654-60.

4. De Vree JM, Ottenhoff R, Bosma PJ, Smith AJ, Aten J, Oude Elferink RP. Correction of liver disease by hepatocyte transplantation in a mouse model of progressive familial intrahepatic cholestasis. Gastroenterology 2000;119:1720-30.

5. Mito M, Kusano M, Kawaura Y. Hepatocyte transplantation in man. Transplant Proc 1992;24:3052-3.

6. Gramignoli R, Vosough M, Kannisto K, Srinivasan RC, Strom SC. Clinical hepatocyte transplantation: practical limits and possible solutions. Eur Surg Res 2015;54:162-77.

7. Meyburg J, Das AM, Hoerster F, et al. One liver for four children: first clinical series of liver cell transplantation for severe neonatal urea cycle defects. Transplantation 2009;87:636-41.

8. Stéphenne X, Najimi M, Sibille C, Nassogne MC, Smets F, Sokal EM. Sustained engraftment and tissue enzyme activity after liver cell transplantation for argininosuccinate lyase deficiency. Gastroenterology 2006;130:1317-23.

9. Mitry RR, Dhawan A, Hughes RD, et al. One liver, three recipients: segment IV from split-liver procedures as a source of hepatocytes for cell transplantation. Transplantation 2004;77:1614-6.

10. Dhawan A, Mitry RR, Hughes RD, et al. Hepatocyte transplantation for inherited factor VII deficiency. Transplantation 2004;78:1812-4.

11. Muraca M, Gerunda G, Neri D, et al. Hepatocyte transplantation as a treatment for glycogen storage disease type 1a. Lancet 2002;359: $317-8$.

12. Sokal EM, Smets F, Bourgois A, et al. Hepatocyte transplantation in a 4year-old girl with peroxisomal biogenesis disease: technique, safety, and metabolic follow-up. Transplantation 2003;76:735-8.

13. Stéphenne X, Debray FG, Smets F, et al. Hepatocyte transplantation using the domino concept in a child with tetrabiopterin nonresponsive phenylketonuria. Cell Transplant 2012;21:2765-70.

14. Beck BB, Habbig S, Dittrich K, et al. Liver cell transplantation in severe infantile oxalosis-a potential bridging procedure to orthotopic liver transplantation? Nephrol Dial Transplant 2012;27:2984-9.

15. Habibullah C, Syed I, Qamar A, Taher-Uz Z. Human fetal hepatocyte transplantation in patients with fulminant hepatic failure. Transplantation 1994;58:951-2.

16. Bilir BM, Guinette D, Karrer F, et al. Hepatocyte transplantation in acute liver failure. Liver Transpl 2000;6:32-40.

17. Khan AA, Habeeb A, Parveen N, et al. Peritoneal transplantation of human fetal hepatocytes for the treatment of acute fatty liver of pregnancy: a case report. Trop Gastroenterol 2004;25:141-3.

18. Schneider A, Attaran M, Meier PN, et al. Hepatocyte transplantation in an acute liver failure due to mushroom poisoning. Transplantation 2006;82:1115-6.

19. Dhawan A, Puppi J, Hughes RD, Mitry RR. Human hepatocyte transplantation: current experience and futurie challenges. Nat Rev Gastroenterol Hepatol 2010;7:288-98.

20. Ibars EP, Cortes M, Tolosa L, et al. Hepatocyte transplantation program: lessons learned and future strategies. World J Gastroenterol. 2016;22: 874-86.

21. Stéphenne X, Najimi M, Ngoc DK, et al. Cryopreservation of human hepatocytes alters the mitochondrial respiratory chain complex 1. Cell Transplant 2007;16:409-19.

22. Wang LJ, Chen YM, George D, et al. Engraftment assessment in human and mouse liver tissue after sex-mismatched liver cell transplantation by real-time quantitative PCR for Y chromosome sequences. Liver Transpl 2002;8:822-8.

23. Tolosa L, Pareja-Ibars E, Teresa Donato M, et al. Neonatal livers: a source for the isolation of good-performing hepatocytes for cell transplantation. Cell Transplant 2014;23:1229-42. 
24. Khan AA, Shaik MV, Parveen N, et al. Human fetal liver-derived stem cell transplantation as supportive modality in the management of end-stage decompensated liver cirrhosis. Cell Transplant 2010;19:409-18.

25. Stangou AJ, Heaton ND, Rela M, Pepys MB, Hawkins PN, Williams R. Domino hepatic transplantation using the liver from a patient with familial amyloid polyneuropathy. Transplantation 1998;65:1496-8.

26. Berry MN, Friend DS. High-yield preparation of isolated rat liver parenchymal cells: a biochemical and fine structural study. J Cell Biol 1969;43:506-20.

27. Seglen PO. Preparation of isolated rat liver cells. Methods Cell Biol 1976;13:29-83.

28. Mitry RR, Hughes RD, Aw MM, et al. Human hepatocyte isolation and relationship of cell viability to early graft function. Cell Transplant 2003;12:69-74.

29. Terry C, Dhawan A, Mitry RR, Lehec SC, Hughes RD. Optimization of the cryopreservation and thawing protocol for human hepatocytes for use in cell transplantation. Liver Transplant 2010;16:229-37.

30. Gramignoli R, Tahan V, Dorko K, et al. Rapid and sensitive assessment of human hepatocyte functions. Cell Transplant 2014;23:1545-56.

31. Rajvanshi P, Kerr A, Bhargava KK, Burk RD, Gupta S. Studies of liver repopulation using the dipeptidyl peptidase IV-deficient rat and other rodent recipients: cell size and structure relationships regulate capacity for increased transplanted hepatocyte mass in the liver lobule. Hepatology 1996;23:482-96.

32. Rajvanshi P, Kerr A, Bhargava KK, Burk RD, Gupta S. Efficacy and safety of repeated hepatocyte transplantation for significant liver repopulation in rodents. Gastroenterology 1996;111:1092-102.

33. Dhawan A. Clinical human hepatocyte transplantation: current status and challenges. Liver Transplant 2015;21:S39-44.

34. Strom SC, Chowdhury JR, Fox IJ. Hepatocyte transplantation for the treatment of human disease. Semin Liver Dis 1999;19:39-48.

35. Jitraruch S, Dhawan A, Hughes RD, et al. Alginate microencapsulated hepatocytes optimised for transplantation in acute liver failure. PLoS ONE 2014;9:e113609.

36. Strom SC, Fisher RA, Rubinstein WS, et al. Transplantation of human hepatocytes. Transplant Proc 1997;29:2103-6.

37. Horslen SP, McCowan TC, Goertzen TC, et al. Isolated hepatocyte transplantation in an infant with a severe urea cycle disorder. Pediatrics 2003;111 (6 Pt 1): 1262-7.

38. Stéphenne X, Najimi M, Smets F, Reding R, De Ville De Goyet J, Sokal EM. Cryopreserved liver cell transplantation controls ornithine transcarbamylase deficient patient while awaiting liver transplantation. Am J Transplant 2005;5:2058-61.

39. Puppi J, Tan N, Mitry RR, et al. Hepatocyte transplantation followed by auxiliary liver transplantation-a novel treatment for ornithine transcarbamylase deficiency. Am J Transpl 2008;8:452-7.

40. Soltys KA, Setoyama K, Tafaleng EN, et al. Host conditioning and rejection monitoring in hepatocyte transplantation in humans. J Hepatol 2017;66:987-1000.

41. Fox IJ, Chowdhury JR, Kaufman SS, et al. Treatment of the Crigler-Najjar syndrome type I with hepatocyte transplantation. N Engl J Med 1998;338: $1422-7$.

42. Ambrosino G, Varotto S, Strom SC, et al. Isolated hepatocyte transplantation for Crigler-Najjar syndrome type 1. Cell Transplant 2005;14:151-7.

43. Lysy P-A, Najimi M, Stephenne X, Bourgois A, Smets F, Sokal E-M. Liver cell transplantation for Crigler-Najjar syndrome type I: update and perspectives. World J Gastroenterol 2008;14:3464-70.

44. Lee K-W, Lee J-H, Shin SW, et al. Hepatocyte transplantation for glycogen storage disease type Ib. Cell Transplant 2007;16:629-37.

45. Grossman M, Rader DJ, Muller DWM, et al. A pilot study of ex vivo gene therapy for homozygous familial hypercholesterolaemia. Nat Med 1995;1: 1148-54.

46. Asonuma K, Gilbert JC, Stein JE, Takeda T, Vacanti JP. Quantitation of transplanted hepatic mass necessary to cure the gunn rat model of hyperbilirubinemia. J Pediatr Surg 1992;27:298-301.
47. Bumgardner GL, Orosz CG. Unusual patterns of alloimmunity evoked by allogeneic liver parenchymal cells. Immunol Rev 2000;174:260-79.

48. Thomson AW, Knolle PA. Antigen-presenting cell function in the tolerogenic liver environment. Nat Rev Immunol 2010;10:753-66.

49. Oldhafer F, Bock M, Falk CS, Vondran FW. Immunological aspects of liver cell transplantation. World J Transplant 2016;6:42.

50. Gupta S, Rajvanshi P, Sokhi R, et al. Entry and integration of transplanted hepatocytes in rat liver plates occur by disruption of hepatic sinusoidal endothelium. Hepatology 1999;29:509-19.

51. Olszewski WL, Interewicz B, Durlik M, Rudowska A, Mecner B. Early loss of transplanted autologous hepatocytes-lysis by leukocytes in vivo and in vitro. Transplant Proc 2001;33:651-3.

52. Gustafson EK, Elgue G, Hughes RD, et al. The instant blood-mediated inflammatory reaction characterized in hepatocyte transplantation. Transplantation 2011;91:632-8.

53. Lee CA, Dhawan A, Smith RA, Mitry RR, Fitzpatrick E. Instant bloodmediated inflammatory reaction in hepatocyte transplantation: current status and future perspectives. Cell Transplant 2016;25:1227-36.

54. Bumgardner GL, Heininger M, Li J, et al. A functional model of hepatocyte transplantation for in vivo immunologic studies. Transplantation 1998;65:53-61.

55. Overturf K, Al-Dhalimy M, Tanguay R, et al. Hepatocytes corrected by gene therapy are selected in vivo in a murine model of hereditary tyrosinaemia type I. Nat Genet 1996;12:266-73.

56. Ding J, Yannam GR, Roy-Chowdhury N, et al. Spontaneous hepatic repopulation in transgenic mice expressing mutant human $\alpha 1$-antitrypsin by wild-type donor hepatocytes. J Clin Invest 2011;121:1930-4.

57. Gupta S. Cell therapy to remove excess copper in Wilson's disease. Ann N Y Acad Sci 2014;1315:70-80.

58. Xiang D, Liu C, Wang M, et al. Non-viral FoxM1 gene delivery to hepatocytes enhances liver repopulation. Cell Death Dis 2014;5:e1252.

59. Jorns C, Nowak G, Nemeth A, et al. De novo donor-specific HLA antibody formation in two patients with Crigler-Najjar Syndrome type I following human hepatocyte transplantation with partial hepatectomy preconditioning. Am J Transplant 2016;16:1021-30.

60. Dagher I, Boudechiche L, Branger J, et al. Efficient hepatocyte engraftment in a nonhuman primate model after partial portal vein embolization. Transplantation 2006;82:1067-73.

61. Puppi J, Strom SC, Hughes RD, et al. Improving the techniques for human hepatocyte transplantation:Report from a consensus meeting in London. Cell Transplant 2012;21:1-10.

62. Mochizuki S, Kawashita Y, Eguchi S, et al. Liver repopulation by transplanted hepatocytes in a rat model of acute liver failure induced by carbon tetrachloride and a partial hepatectomy. Ann Transpl 2010;15: 49-55.

63. Irani AN, Malhi H, Slehria S, et al. Correction of liver disease following transplantation of normal rat hepatocytes into Long-Evans Cinnamon rats modeling Wilson's disease. Mol Ther 2001;3:302-9.

64. Abdalla EK. Portal vein embolization (prior to major hepatectomy) effects on regeneration, resectability, and outcome. J Surg Oncol 2010;102:960-7.

65. Dagher I, Nguyen TH, Groyer-Picard MT, et al. Efficient hepatocyte engraftment and long-term transgene expression after reversible portal embolization in nonhuman primates. Hepatology 2009;49:950-9.

66. Goulinet-Mainot S, Tranchart H, Groyer-Picard MT, et al. Improved hepatocyte engraftment after portal vein occlusion in LDL receptordeficient WHHL rabbits and lentiviral-mediated phenotypic correction in vitro. Cell Med 2012;4:85-98.

67. Zhou H, Dong X, Kabarriti R, et al. Single liver lobe repopulation with wildtype hepatocytes using regional hepatic irradiation cures jaundice in Gunn rats. PLoS ONE 2012;7:e46775.

68. Ashokkumar C, Bentlejewski C, Sun Q, et al. Allospecific CD154+ B cells associate with intestine allograft rejection in children. Transplantation 2010;90:1226-31.

69. Ashokkumar C, Talukdar A, Sun Q, et al. Allospecific CD154+ T cells associate with rejection risk after pediatric liver transplantation. Am J Transplant 2009;9:179-91. 
70. Bohnen NI, Charron M, Reyes J, et al. Use of indium-111-labeled hepatocytes to determine the biodistribution of transplanted hepatocytes through portal vein infusion. Clin Nucl Med 2000;25:447-50.

71. Puppi J, Mitry RR, Modo M, Dhawan A, Raja K, Hughes RD. Use of a clinically approved iron oxide MRI contrast agent to label human hepatocytes. Cell Transplant 2011;20:963-75.

72. Raschzok N, Teichgräber U, Pratschke J, Sauer I. Preclinical swine models for monitoring of hepatocyte transplantation by MRI. Methods Mol Biol 2017;1506:201-14.

73. Mas VR, Maluf DG, Thompson M, Ferreira-Gonzalez A, Fisher RA. Engraftment measurement in human liver tissue after liver cell transplantation by short tandem repeats analysis. Cell Transplant 2004;13:231-6.

74. Vallabhajosyula P, Korutla L, Habertheuer A, et al. Tissue-specific exosome biomarkers for noninvasively monitoring immunologic rejection of transplanted tissue. J Clin Invest 2017;127:1-17.

75. Schütz E, Fischer A, Beck J, et al. Graft-derived cell-free DNA, a noninvasive early rejection and graft damage marker in liver transplantation: a prospective, observational, multicenter cohort study. PLoS Med 2017;14:1-19.

76. Nicolas C, Wang Y, Luebke-Wheeler J, Nyberg S. Stem cell therapies for treatment of liver disease. Biomedicines 2016;4:2.

77. Najimi M, Khuu DN, Lysy PA, et al. Adult-derived human liver mesenchymal-like cells as a potential progenitor reservoir of hepatocytes? Cell Transplant 2007;16:717-28.
78. Wu X-B, Tao R. Hepatocyte differentiation of mesenchymal stem cells. Hepatobiliary Pancreat Dis Int 2012;11:360-71.

79. Iyer SS, Rojas M. Anti-inflammatory effects of mesenchymal stem cells: novel concept for future therapies. Expert Opin Biol Ther 2008;8: 569-81.

80. Fitzpatrick E, Wu Y, Dhadda P, et al. Co-culture with mesenchymal stem cells results in improved viability and function of human hepatocytes. Cell Transplant 2013;24:1-29.

81. Roy-Chowdhury N, Wang X, Guha C, Roy-Chowdhury J. Hepatocyte-like cells derived from induced pluripotent stem cells. Hepatol Int 2016;11: 1-16.

82. Baxter M, Withey S, Harrison S, et al. Phenotypic and functional analyses show stem cell-derived hepatocyte-like cells better mimic fetal rather than adult hepatocytes. J Hepatol 2015;62:581-9.

83. Hannoun Z, Steichen C, Dianat N, Weber A, Dubart-Kupperschmitt A. The potential of induced pluripotent stem cell derived hepatocytes. J Hepatol 2016;65:182-99.

84. Hickey RD, Mao SA, Glorioso J, et al. Curative ex vivo liver-directed gene therapy in a pig model of hereditary tyrosinemia type 1. Sci Transl Med 2016;8:1-10.

85. Mitry RR, Jitraruch S, Iansante V, Dhawan A. Alginate encapsulation of human hepatocytes and assessment of microbeads. Methods Mol Biol 2017: 273-81.

86. Jitraruch S, Dhawan A, Hughes RD, et al. Cryopreservation of hepatocyte microbeads for clinical transplantation. Cell Transplant 2017;26:1341-54. 